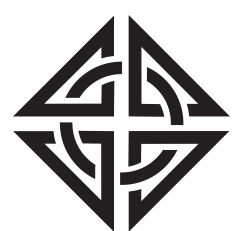

SCIENTIA
Sharif University of Technology

Scientia Iranica

Transactions A: Civil Engineering

http://scientiairanica.sharif.edu

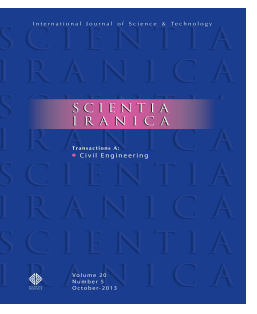

\title{
An exact solution to the problems of flexo-poroelastic structures rested on elastic beds acted upon by moving loads
}

\author{
A. Nikkhoo ${ }^{\mathrm{a}}$, R. Tafakor ${ }^{\mathrm{a}}$, and M. Mofid ${ }^{\mathrm{b}, *}$ \\ a. Department of Civil Engineering, University of Science and Culture, Tehran, Iran. \\ b. Department of Civil Engineering, Sharif University of Technology, Tehran, Iran.
}

Received 3 July 2018; received in revised form 23 September 2018; accepted 26 January 2019

\section{KEYWORDS}

Poroelastic beam-like structures;

Dynamical analysis;

Moving loads;

Laplace transform

method;

Analytical approach.

\begin{abstract}
This paper aims to examine flexural vibrations of fully saturated poroelastic structures on an elastic bed subjected to moving point loads via an analytical solution. Using a flexural beam model in conjunction with Biot's poroelasticity theory, the equations of motion of the porous structure were derived. By using the assumed mode method and Laplace transform, the explicit expressions of displacement and pore pressure were obtained carefully. For a particular case, the predicted results were compared with those of another work and thus, reasonably good agreement was achieved. The effects of the moving load velocity, permeability ratio, transverse stiffness of the foundation, viscosity of the pore fluid, and porosity on the maximum elasto-dynamic fields and pore pressure were conclusively discussed. The velocity pertinent to the maximum possible dynamic response was graphically determined and the effects of influential parameters on this crucial factor were displayed. The present model could be easily extended to multi-layered poroelastic structures under moving loads.
\end{abstract}

(C) 2020 Sharif University of Technology. All rights reserved.

\section{Introduction}

Based on Terzaghi's consolidation theory [1], Biot [2-5] initiated the continuum mechanics theory of dynamic poroelastic bodies. This theory deals with the motion of both solid and fluid within its pores and their interactions [6,7]. In general, the fluid phase consists of both liquid and gas matters. A poroelastic body is characterized by its porosity, permeability, and the

*. Corresponding author. Tel.: +982166164214;

Fax: +982166014828

E-mail addresses: nikkhoo@usc.ac.ir (A. Nikkhoo);

r.tafakor@usc.ac.ir (R.Tafakor); mofid@sharif.edu (M. Mofid)

doi: $10.24200 /$ sci.2019.51365.2135 properties of its constituents, namely solid skeleton and fluid. It is implied that all these data should be appropriately incorporated into the equations of motion of the porous structure. For many practical applications, the linear theory of poroelasticity can be safely applied to stress analysis of porous media. To date, such a theory has been widely applied to a large body of problems pertinent to biomechanics and medical engineering [8-10], geomechanics [1113], hydrology [14,15], materials science [16-18], and physics and geophysics [19-22].

Concerning the mechanical behavior of a poroelastic structure subjected to dynamic load, typical behavior of time-varying pore pressure was initially reported by Mandel [23]. An analytical solution to threedimensional consolidation problems based on Biot's 
theory [2] was proposed, and the non-monotonic pore water pressure was observed. Afterward, Cryer [24] predicted similar results for consolidation of the center of a porous sphere zone acted upon by a hydrostatic pressure. The influence of Poisson's ratio on the pore pressure was also addressed. Such a non-monotonic response of the pore fluid pressure was then referred to as the Mandel-Cryer effect [25,26]. Abousleiman et al. [27] generalized Mandel's problem to a poroelastic medium with compressible fluid and transverse isotropy. Further, a full solution of stresses, displacements, and pore pressure was obtained to display the Mandel-Cryer effect. Until now, poroelastic beams have been widely employed as simple models for structural elements, bone structures, and geological layers. Li et al. [28] developed the equations of motion for a transversely isotropic poroelastic beam subjected to longitudinal or transverse loads when only the longitudinal motion of the fluids within the pores was possible. Using series solutions, the quasi-static problem of the porous beam was examined in different mechanical and diffusion boundary conditions. Cederbaum et al. [29,30] found interesting behavior patterns of poroelastic beams and columns with only longitudinal diffusion. In this paper, based on the developed poroelastic model by $\mathrm{Li}$ et al. [28], transverse vibration of a porous beam-like structure subjected to a moving concentrated load is selected to be investigated in some detail.

Vibrations of solid beam and plate structures acted upon by moving loads [31-37], moving masses [38-54], and moving mass-sprung systems [5557] have already been examined. Kiani et al. [58] examined the effect of shear deformation on the vibrational behavior of poroelastic beams under a moving pointed load. Most of these studies displayed the influence of the dynamical parameters of the system on the transverse displacement of the elastic beam. On the other hand, dynamic responses of poroelastic half-space soil media subjected to moving loads have been extensively studied [59-66]. In most of these investigations, the time history plots of transverse displacement and pore pressure of particular points were demonstrated for special levels of the velocity. Additionally, the effect of the moving load velocity on the maximum dynamic response of displacements and pore pressure was not explained and discussed. Such an important issue plays a vital role in the optimal design and practical applications of porous structures. Herein, the porous medium traversed by a moving load is modeled by a poroelastic beam of finite length, and determination of the effects of the foundation stiffness, permeability, and velocity of the moving pointed load on the maximum transverse displacement and maximum pore pressure is of particular interest.

To date, flexural vibrations of poroelastic plates have been examined in some detail [67-71]. Further, in- plane and torsional vibrations of poroelastic cylinders have been studied [72,73]. Although there exists plentiful literature of solutions on different classes of problems, an analytical solution for the vibration of poroelastic beams acted upon by a moving load remains hitherto absent. Thereby, we focus on a class of poroelastic beam-like structures with only longitudinal diffusion of fluid within the pore network. Since only fluid movement in the longitudinal direction is permitted, it is expected that transverse vibration of the structure could be controlled more effectively by not only the mechanical boundary conditions, but also by the diffusion conditions at both ends of the poroelastic beam. The present work deals with an exemplifying model to explore the vibration of saturated poroelastic media, confined between doubly parallel impermeable layers, under moving loads. For example, a confined soil layer via geotextile layers subjected to moving vehicles can be appropriately modeled in this paper.

In the present work, transverse vibration of beamlike poroelastic structures resting on an elastic foundation due to a moving point load is of concern. By employing Biot's and Euler-Bernoulli beam theories, coupled equations of motion are developed and analytically solved. The explicit expressions of dynamic displacement and pore pressure of a simply supported poroelastic beam are derived. Through different numerical studies, the effects of the influential factors on the maximum values of displacement and pore pressure are investigated. The obtained results of poroelastic beams with low and high levels of permeability are discussed with respect to a wide range of the velocities of the moving load.

\section{Basic assumptions and formulations}

Consider a homogeneous poroelastic beam fully saturated by a fluid that can only move along the longitudinal direction of the beam, as shown in Figure 1. The density of the fully saturated medium, length, moment inertia, and cross-sectional area of the beam are denoted by $\rho, l, I$, and $A$, respectively. The beam

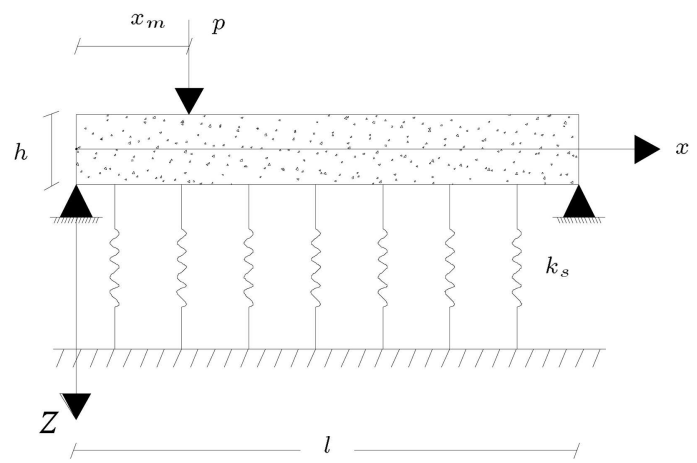

Figure 1. A poroelastic beam-like structure with simple-permeable ends. 
subjected to a load moves at a constant velocity, $v$. The moving load enters the beam from the left side at time $t=0$ and its location on its upper surface is represented by $x_{m}$. The poroelastic beam is located on an elastic medium. To consider the transverse interaction of the beam and its foundation, the beam remains continuously attached to an elastic layer with constant $k_{s}$ (i.e., Winkler spring model). It is assumed that the height of the beam is fairly negligible in comparison to its length. Further, after deformation by the moving point load, each cross-sectional plane remains plane which is perpendicular to the neutral axis. Therefore, the beam could be rationally modeled based on the Euler-Bernoulli beam theory. Based on Biot's model [2] for transversely isotropic materials, the constitutive equations are read as follows:

$$
\begin{aligned}
& \left\{\begin{array}{l}
\sigma_{x x}^{s} \\
\sigma_{y y}^{s} \\
\sigma_{z z}^{s}
\end{array}\right\}=\left[\begin{array}{lll}
c_{11} & c_{12} & c_{12} \\
c_{12} & c_{22} & c_{23} \\
c_{12} & c_{23} & c_{22}
\end{array}\right]\left\{\begin{array}{l}
\epsilon_{x x}^{s} \\
\epsilon_{y y}^{s} \\
\epsilon_{z z}^{s}
\end{array}\right\}-\left\{\begin{array}{l}
\alpha_{1} \\
\alpha_{2} \\
\alpha_{2}
\end{array}\right\} p_{f}, \\
& p_{f}=G\left(\zeta-\alpha_{1} \epsilon_{x x}^{s}-\alpha_{2}\left(\epsilon_{y y}^{s}+\epsilon_{z z}^{s}\right)\right),
\end{aligned}
$$

where $\sigma_{\alpha \alpha}^{s}$ represents the effective normal stress, $\epsilon_{\alpha \alpha}^{s}$ the normal strains of the skeleton, $c_{i j}$ the material property matrix, $\zeta$ the pore volume change, and $p_{f}$ the pore pressure. The parameters $\alpha_{i}$ and $G$ rely on the properties of both solid and fluid phases as well as the morphology of the pores. The two latter ones link the deformation of the solid phase to the movement of fluid inside the pores, and vice versa. Since the bending behavior of the poroelastic medium subjected to a moving load is of interest, one can rationally set $\sigma_{y y}^{s}=\sigma_{z z}^{s}=0$. As a result, the constitutive equations in Eqs. (1a) and (1b) are reduced to the following relations:

$$
\begin{gathered}
\sigma_{x x}^{s}=E \epsilon_{x x}^{s}-\eta p_{f}, \\
\zeta=\eta \epsilon_{x x}^{s}+\beta p_{f},
\end{gathered}
$$

where $E, \eta$, and $\beta$ are functions of $c_{i j}, \alpha_{i}$, and $G$. The parameter $E$ exactly represents the longitudinal elastic modulus of the poroelastic beam. According to the hypothesis of the Euler-Bernoulli beam, in the absence of the longitudinal deformation of the neutral axis, the longitudinal displacement of the beam is read as $u_{x}=-z \frac{\partial w}{\partial x}$, where $\partial$ denotes the partial derivative sign and $w=w(x, t)$ is the transverse displacement field of the poroelastic beam.

Thereby, $\epsilon_{x x}^{s}=\frac{\partial u_{x}}{\partial x}=-z \frac{\partial^{2} w}{\partial x^{2}}$. Now, let's define bending moment of the solid phase by: $M=$ $\int_{A} \sigma_{x x}^{s} \mathrm{~d} A$. By substituting Eq. (2a) as a function of transverse displacement into the recent relation, the bending moment within the poroelastic beam is obtained as follows:

$$
M=-E I \frac{\partial^{2} w}{\partial x^{2}}+\eta M_{p} ; \quad M_{p}=-\int_{A} z p_{f} \mathrm{~d} A
$$

where $M_{p}$ is defined as the pore pressure moment. Using Newton's second law for an element of the poroelastic beam by neglecting the rotary inertia effect, the governing equations could be expressed as follows:

$$
\begin{aligned}
& Q=\frac{\partial M}{\partial x}, \\
& \frac{\partial Q}{\partial x}=k_{s} w-P \delta\left(x-x_{m}\right) H\left(l-x_{m}\right)+\rho A \frac{\partial^{2} w}{\partial t^{2}},
\end{aligned}
$$

where $t, H$, and $\delta$ represent the time parameter, Heaviside step function, and Dirac delta function, respectively. Substituting Eq. (4a) into Eq. (4b) and then, introducing Eq. (3) to the resulting equation, we get:

$$
\begin{gathered}
\rho A \frac{\partial^{2} w}{\partial t^{2}}+E I \frac{\partial^{4} w}{\partial x^{4}}-\eta \frac{\partial^{2} M_{p}}{\partial x^{2}}+k_{s} w \\
=P \delta\left(x-x_{m}\right) H\left(l-x_{m}\right) .
\end{gathered}
$$

Eq. (5) depicts the transverse vibration of a poroelastic beam with the longitudinal movement of the fluid subjected to a moving load. This equation is essentially constructed on the basis of the Euler-Bernoulli beam model. It indicates that to obtain the displacement field of the poroelastic beam with good accuracy, the skeleton phase should satisfy the hypotheses of such a beam theory with sufficient accuracy. For capturing the dynamic response of thick enough porous beams or those with low levels of the slenderness ratio, shear deformable beam theories should be employed [7476]. Additionally, the present equation only covers the case that the fluid inside the pores only moves along the longitudinal direction and its transverse seepage is prohibited. Otherwise, the interactions between deformation of the beam and transverse movement of the fluid must be also taken into account. The discussion on such interesting subjects is out of the scope of the present research and could be considered as hot topics for future complementary works.

Concerning the movement of the fluid through the connected pores within the beam, the generalized form of Darcy's law by Biot [2] could be employed as follows:

$$
\frac{\partial \mathbf{d}}{\partial t}=-\frac{1}{\mu_{f}} \mathbf{k}_{p} \nabla p_{f}
$$

where $\mathbf{d}=\phi\left(\mathbf{u}^{s}-\mathbf{u}^{f}\right), \phi$ is the porosity, $\mathbf{u}^{s}$ and $\mathbf{u}^{f}$ in order are the displacement vectors of the solid and fluid phases, $\mu_{f}$ is the fluid viscosity, $\mathbf{k}_{p}$ denotes the permeability tensor, and $\nabla$ is the gradient sign. Since $\zeta=-\nabla . d$ and only the movement of the fluid along the longitudinal direction of the beam is possible, one can obtain the following:

$$
\frac{\partial \zeta}{\partial t}=\frac{k_{p}}{\mu_{f}} \frac{\partial^{2} p_{f}}{\partial x^{2}}
$$


where $k_{p}$ is the longitudinal permeability of the porous medium. By substituting Eq. (2b) into Eq. (7), premultiplying both sides of the resulting equation by $-z$ and taking the integral from both sides over the cross-sectional area of the poroelastic beam yield:

$$
\frac{\partial M_{p}}{\partial t}-k \frac{\partial^{2} M_{p}}{\partial x^{2}}+\lambda E I \frac{\partial^{3} w}{\partial t \partial x^{2}}=0
$$

where $k=\frac{\lambda E k_{p}}{\eta \mu_{f}}$ and $\lambda=\frac{\eta}{\beta E}$. Eq. (8) explains longitudinal movement of the fluid inside the pores of the poroelastic medium in terms of pore pressure moment. It can be readily proved that for the poroelastic beam under study, $p_{f}=-\frac{M_{p} z}{I}$. It implies that there exists a linear relationship between the pore pressure and its moment at each point of the poroelastic beam.

Eqs. (5) and (8) represent the fundamental coupled equations of a transversely vibrating poroelastic beam subjected to a moving load. In order to determine the unknown fields of the problem, namely $w$ and $M_{p}$, the appropriate initial and boundary conditions should be imposed. It is assumed that the beam has simple ends and the seepage can freely occur at its ends. Therefore, the fields of our interest should satisfy the following boundary conditions:

$$
\begin{aligned}
& w(0, t)=w(l, t)=0 ; \quad M(0, t)=M(l, t)=0, \\
& M_{p}(0, t)=M_{p}(l, t)=0 .
\end{aligned}
$$

Additionally, the poroelastic beam is assumed to be at rest at the entrance of the moving load. Thereby, the following initial conditions should be imposed:

$$
w(x, 0)=0, \quad \frac{\partial w}{\partial t}(x, 0)=0 ; \quad M_{p}(x, 0)=0 .
$$

To evaluate the elastic fields of the porous medium, Eqs. (5) and (8) with the given conditions in Eqs. (9) and (10) should be appropriately solved. In the next part, an analytical solution is developed for dynamic analysis of the poroelastic beam under excitation of a moving point load.

\section{Development of an analytical solution}

To analyze the problem in a more general framework, the following dimensionless parameters are considered:

$$
\begin{array}{lll}
x^{*}=\frac{x}{l}, & x_{m}^{*}=\frac{x_{m}}{l}, & w^{*}=\frac{w}{l}, \\
\tau=\frac{t}{l^{2}} \sqrt{\frac{E I}{\rho A}}, & k_{s}^{*}=\frac{k_{s} l^{4}}{E I}, & M_{p}^{*}=\frac{M_{p} l}{E I}, \\
P^{*}=\frac{P l^{2}}{E I}, & k^{*}=\frac{1}{k} \sqrt{\frac{E I}{\rho A} .} &
\end{array}
$$

By introducing Eq. (11) to Eqs. (5) and (8), the dimensionless governing equations of the problem at hand take the following form:

$$
\begin{gathered}
\frac{\partial^{2} w^{*}}{\partial \tau^{2}}+\frac{\partial^{4} w^{*}}{\partial x^{* 4}}-\eta \frac{\partial^{2} M_{p}^{*}}{\partial x^{* 2}}+k_{s}^{*} w^{*} \\
=P^{*} \delta\left(x^{*}-x_{m}^{*}\right) H\left(1-x_{m}^{*}\right), \\
k^{*} \frac{\partial M_{p}^{*}}{\partial \tau}-\frac{\partial^{2} M_{p}^{*}}{\partial x^{* 2}}+\lambda k^{*} \frac{\partial^{3} w^{*}}{\partial \tau \partial x^{* 2}}=0,
\end{gathered}
$$

with the following boundary and initial conditions:

$$
\begin{aligned}
& w^{*}(0, \tau)=w^{*}(1, \tau)=0, \\
& \frac{\partial^{2} w^{*}}{\partial^{2} x^{*}}(0, \tau)=\frac{\partial^{2} w^{*}}{\partial^{2} x^{*}}(1, \tau)=0, \\
& M_{p}^{*}(0, \tau)=M_{p}^{*}(1, \tau)=0, \\
& w^{*}\left(x^{*}, 0\right)=0, \quad \frac{\partial w^{*}}{\partial \tau}\left(x^{*}, 0\right)=0, \\
& M_{p}^{*}\left(x^{*}, 0\right)=0 .
\end{aligned}
$$

Using the assumed mode method, the dimensionless transverse displacement and pore pressure moment of the poroelastic beam can be expressed in terms of admissible mode shapes as follows:

$$
\begin{aligned}
& w^{*}\left(x^{*}, \tau\right)=\sum_{n=1}^{\infty} a_{n}(\tau) \sin \left(n \pi x^{*}\right), \\
& M_{p}^{*}\left(x^{*}, \tau\right)=\sum_{n=1}^{\infty} b_{n}(\tau) \sin \left(n \pi x^{*}\right),
\end{aligned}
$$

where $a_{n}(\tau)$ and $b_{n}(\tau)$ are the time-dependent parameters that should be determined. In the remainder of this section, at two time intervals, analytical solutions to the problem based on the Laplace transform are given:

Phase I: When the moving load is being in contact with the base beam: $0<\tau<\tau_{f}$. By substituting Eqs. (14a) and (14b) into Eqs. (12a) and (12b) and using the relation:

$$
\delta\left(x^{*}-x_{m}^{*}\right)=2 \sum_{n=1}^{\infty} \sin \left(n \pi x_{m}^{*}\right) \sin \left(n \pi x^{*}\right),
$$

the following linear Ordinary Differential Equations (ODEs) are obtained:

$$
\begin{aligned}
& \frac{\mathrm{d}^{2} a_{n}}{\mathrm{~d} \tau^{2}}+\left((n \pi)^{4}+k_{s}^{*}\right) a_{n}+\eta(n \pi)^{2} b_{n}=2 P^{*} \sin \left(g_{n}^{*} \tau\right) \\
& \frac{k^{*}}{(n \pi)^{2}} \frac{\mathrm{d} b_{n}}{\partial \tau}-\lambda k^{*} \frac{\mathrm{d} a_{n}}{\partial \tau}+b_{n}=0
\end{aligned}
$$

with the following initial values: 


$$
a_{n}(0)=b_{n}(0)=0, \quad \frac{\mathrm{d} a_{n}(0)}{\mathrm{d} \tau}=\frac{\mathrm{d} b_{n}(0)}{\mathrm{d} \tau}=0
$$

where $g_{n}^{*}=n \pi v l \sqrt{\frac{E I}{\rho A}}$. By taking the Laplace transform of both sides of Eqs. (16a) and (16b):

$$
\begin{aligned}
& \mathcal{L}\left\{a_{n}\right\}=\frac{A_{1_{n}}+A_{2_{n}} s}{\sum_{m=0}^{5} R_{m_{n}} s^{m}}, \\
& \mathcal{L}\left\{b_{n}\right\}=\frac{B_{1_{n}} s}{\sum_{m=0}^{5} R_{m_{n}} s^{m}},
\end{aligned}
$$

where:

$$
\begin{aligned}
& A_{1_{n}}=2 P^{*} g_{n}^{*}, \quad A_{2_{n}}=2 P^{*} g_{n}^{*}(n \pi)^{2} / k^{*}, \\
& B_{1_{n}}=2 P^{*} g_{n}^{*} \lambda(n \pi)^{2}, \quad R_{5_{n}}=1, \\
& R_{4_{n}}=\frac{(n \pi)^{2}}{k^{*}}, \quad R_{3_{n}}=g_{n}^{* 2}+k_{s}^{*}+(n \pi)^{4}(1+\lambda \eta), \\
& R_{2_{n}}=\frac{(n \pi)^{2}}{k^{*}}\left(\Gamma_{n}^{2}+g_{n}^{* 2}\right), \\
& R_{1_{n}}=g_{n}^{* 2}\left(k_{s}^{*}+(n \pi)^{4}(1+\lambda \eta)\right), \\
& R_{0_{n}}=\frac{g_{n}^{* 2}(n \pi)^{2}}{k^{*}} \Gamma_{n}^{2}, \quad \Gamma_{n}^{2}=(n \pi)^{4}+k_{s}^{*} .
\end{aligned}
$$

Eqs. (18a) and (18b) can be also rewritten in terms of fractional statements from which inverse Laplace transform could be evaluated more conveniently. Hence:

$$
\begin{aligned}
& \mathcal{L}\left\{a_{n}\right\}=\sum_{i=1}^{5} \frac{\Re\left(A_{i_{n}}^{\prime}\right)+\mathrm{i} \Im\left(A_{i_{n}}^{\prime}\right)}{s-p_{i_{n}}^{\prime}}, \\
& \mathcal{L}\left\{b_{n}\right\}=\sum_{i=1}^{5} \frac{\Re\left(B_{i_{n}}^{\prime}\right)+\mathrm{i} \Im\left(B_{i_{n}}^{\prime}\right)}{s-p_{i_{n}}^{\prime}},
\end{aligned}
$$

where $p_{i_{n}}^{\prime}(i=1, \cdots, 5)$ are the complex roots of the fifth-order polynomial of the denominators of Eq. (18) (which is also called the characteristic relation of the poroelastic beam), and the procedure of calculating the complex numbers $A_{i_{n}}^{\prime}$ and $B_{i_{n}}^{\prime}$ has been explained in some detail in Appendix A1. Thereby, by applying the inverse Laplace to both Eqs. (20a) and (20b), the dimensionless dynamic transverse displacement and pore pressure moment of the poroelastic beam during the course of excitation are provided by:

$$
\begin{aligned}
w^{*}\left(x^{*}, \tau\right)= & \sum_{n=1}^{\infty} \sum_{i=1}^{5}\left(\Re\left(A_{i_{n}}^{\prime}\right)+\mathrm{i} \Im\left(A_{i_{n}}^{\prime}\right)\right) \\
& \exp \left(p_{i_{n}}^{\prime} \tau\right) \sin \left(n \pi x^{*}\right), \\
M_{p}^{*}\left(x^{*}, \tau\right)= & \sum_{n=1}^{\infty} \sum_{i=1}^{5}\left(\Re\left(B_{i_{n}}^{\prime}\right)+\mathrm{i} \Im\left(B_{i_{n}}^{\prime}\right)\right) \\
& \exp \left(p_{i_{n}}^{\prime} \tau\right) \sin \left(n \pi x^{*}\right) .
\end{aligned}
$$

Phase II: When the moving load has left the poroelastic beam: $\tau>\tau_{f}$. In this case, by introducing Eqs. (14a) and (14b) to Eqs. (12a) and (12b), the governing equations associated with the free vibration of the poroelastic beam in terms of time-dependent parameters are derived as follows:

$$
\begin{aligned}
& \frac{\mathrm{d}^{2} a_{n}}{\mathrm{~d} \tau^{\prime 2}}+\left((n \pi)^{4}+k_{s}^{*}\right) a_{n}+\eta(n \pi)^{2} b_{n}=0 ; \quad \begin{array}{r}
\tau>\tau_{f}, \\
\frac{k^{*}}{(n \pi)^{2}}
\end{array} \frac{\mathrm{d} b_{n}}{\mathrm{~d} \tau^{\prime}}-\lambda k^{*} \frac{\mathrm{d} a_{n}}{\mathrm{~d} \tau^{\prime}}+b_{n}=0,
\end{aligned}
$$

where $a_{n}=a_{n}\left(\tau^{\prime}\right), b_{n}=b_{n}\left(\tau^{\prime}\right)$, and $\tau^{\prime}=\tau-\tau_{f}$. Additionally, the requirement of continuity of the transverse displacement and pore pressure moment as well as their velocities at the end of the excitation phase (i.e., $\tau=\tau_{f}$ ) leads to the following initial conditions:

$$
\begin{aligned}
& a_{n}\left(\tau^{\prime}=0\right)=a_{n}\left(\tau_{f}\right) \\
& =\sum_{i=1}^{5}\left(\Re\left(A_{i_{n}}^{\prime}\right)+\mathrm{i} \Im\left(A_{i_{n}}^{\prime}\right)\right) \exp \left(p_{i_{n}}^{\prime} \tau_{f}\right), \\
& b_{n}\left(\tau^{\prime}=0\right)=b_{n}\left(\tau_{f}\right) \\
& =\sum_{i=1}^{5}\left(\Re\left(B_{i_{n}}^{\prime}\right)+\mathrm{i} \Im\left(B_{i_{n}}^{\prime}\right)\right) \exp \left(p_{i_{n}}^{\prime} \tau_{f}\right), \\
& \frac{\partial a_{n}}{\partial \tau}\left(\tau^{\prime}=0\right)=\frac{\partial a_{n}}{\partial \tau}\left(\tau=\tau_{f}\right) \\
& \quad=\sum_{i=1}^{5} p_{i_{n}}^{\prime}\left(\Re\left(A_{i_{n}}^{\prime}\right)+\mathrm{i} \Im\left(A_{i_{n}}^{\prime}\right)\right) \exp \left(p_{i_{n}}^{\prime} \tau_{f}\right), \\
& \frac{\partial b_{n}}{\partial \tau}\left(\tau^{\prime}=0\right)=\frac{\partial b_{n}}{\partial \tau}\left(\tau=\tau_{f}\right) \\
& \quad=\sum_{i=1}^{5} p_{i_{n}}^{\prime}\left(\Re\left(B_{i_{n}}^{\prime}\right)+\mathrm{i} \Im\left(B_{i_{n}}^{\prime}\right)\right) \exp \left(p_{i_{n}}^{\prime} \tau_{f}\right) \cdot(23)
\end{aligned}
$$

By employing Laplace transform to solve the set of ODEs in Eqs. (22a) and (22b) with the given initial values in Eq. (23), one may arrive at:

$$
\begin{aligned}
& \mathcal{L}\left\{a_{n}\right\}=\sum_{m=0}^{2} A_{m_{n}}^{\prime} s^{m} / \sum_{m=0}^{3} r_{m_{n}} s^{m}, \\
& \mathcal{L}\left\{b_{n}\right\}=\sum_{m=0}^{3} B_{m_{n}}^{\prime} s^{m} / \sum_{m=0}^{4} r_{m_{n}}^{\prime} s^{m},
\end{aligned}
$$

where:

$$
A_{2_{n}}^{\prime}=a_{n}(0), \quad B_{3_{n}}^{\prime}=b_{n}(0),
$$




$$
\begin{aligned}
& A_{1_{n}}^{\prime}=\frac{(n \pi)^{2}}{k^{*}} a_{n}(0)+\frac{\mathrm{d} a_{n}}{\mathrm{~d} \tau^{\prime}}(0), \\
& B_{2_{n}}^{\prime}=(n \pi)^{2}\left(\lambda \frac{\mathrm{d} a_{n}}{\mathrm{~d} \tau^{\prime}}(0)+\frac{b_{n}(0)}{k^{*}}\right), \\
& A_{0_{n}}^{\prime}=\frac{\mathrm{d} a_{n}}{\mathrm{~d} \tau^{\prime}}(0) \frac{(n \pi)^{2}}{k^{*}}+\lambda \eta(n \pi)^{4} a_{n}(0)-\eta(n \pi)^{2} b_{n}(0), \\
& B_{1_{n}}^{\prime}=\lambda(n \pi)^{2}\left(\frac{(n \pi)^{2}}{k^{*}} \frac{\mathrm{d} a_{n}}{\mathrm{~d} \tau^{\prime}}(0)-a_{n}(0) \Gamma_{n}^{2}\right)+b_{n}(0) \Gamma_{n}^{2}, \\
& B_{0_{n}}^{\prime}=\frac{\left(n \pi \Gamma_{n}\right)^{2}}{k^{*}}\left(b_{n}(0)-\lambda(n \pi)^{2} a_{n}(0)\right), \\
& r_{3_{n}}=1, \quad r_{4_{n}}^{\prime}=1, \quad r_{2_{n}}=\frac{(n \pi)^{2}}{k^{*}}, \\
& r_{3_{n}}^{\prime}=\frac{2(n \pi)^{2}}{k^{*}}, \quad r_{1_{n}}^{\prime}=\frac{\Gamma_{n}^{2}(n \pi)^{4}}{k^{* 2}}(25) \\
& r_{2_{n}}^{\prime}=\Gamma_{n}^{2}+\lambda \eta(n \pi)^{4}+\frac{(n \pi)^{4}}{k^{* 2}}, \quad \lambda \eta(n \pi)^{4}, \\
& k^{*} \Gamma_{n}^{*}
\end{aligned}
$$

Now, by decomposing Eqs. (24a) and (24b) into simpler ratios and taking the inverse Laplace transform of the resulting expressions, the dynamic transverse displacement and pore pressure moment of the poroelastic beam during the course of free vibration are evaluated as follows:

$$
\begin{aligned}
w^{*}\left(x^{*}, \tau^{\prime}\right)= & \sum_{n=1}^{\infty} \sum_{i=1}^{3}\left(\Re\left(A_{i_{n}}^{\prime \prime}\right)+\mathrm{i} \Im\left(A_{i_{n}}^{\prime \prime}\right)\right) \\
& \exp \left(p_{i_{n}}^{\prime \prime} \tau^{\prime}\right) \sin \left(n \pi x^{*}\right), \\
M_{p}^{*}\left(x^{*}, \tau^{\prime}\right)= & \sum_{n=1}^{\infty} \sum_{i=1}^{4}\left(\Re\left(B_{i_{n}}^{\prime \prime}\right)+\mathrm{i} \Im\left(B_{i_{n}}^{\prime \prime}\right)\right) \\
& \exp \left(q_{i_{n}}^{\prime \prime} \tau^{\prime}\right) \sin \left(n \pi x^{*}\right),
\end{aligned}
$$

where the values of $A_{i_{n}}^{\prime \prime}, B_{i_{n}}^{\prime \prime}, p_{i_{n}}^{\prime \prime}$ and $q_{i_{n}}^{\prime \prime}$ are given in Appendix A2.

\section{Results and discussion}

\subsection{Some comparative studies in particular cases}

To check the validity of the conducted calculations, the results obtained by the proposed model are compared with those of other works in some particular cases.
Lee [77] studied the dynamic response of a Timoshenko beam on an elastic foundation due to moving mass. The effects of the velocity of the moving mass as well as the transverse stiffness of the foundation on the dynamic response were examined. In the first comparative study, consider an elastic beam with the following properties:

$$
\begin{aligned}
& E=207 \times 10^{9} \mathrm{~N} / \mathrm{m}^{2}, \\
& \rho=7700 \mathrm{~kg} / \mathrm{m}^{3}, \\
& \beta^{\prime}=0.03, \\
& l=1 \mathrm{~m} .
\end{aligned}
$$

Further, we define:

$$
\begin{aligned}
& \beta^{\prime}=\frac{\pi r_{0}}{l}, \\
& V_{c r}=\frac{\pi}{l} \sqrt{\frac{E I}{\rho A}}, \\
& U_{N}\left(x_{m}, t\right)=\frac{u\left(x_{m}, t\right)}{\delta},
\end{aligned}
$$

where $r_{0}$ and $\delta$ respectively denote the gyration radius of the beam's cross-section and the static deflection of the beam's midspan point due to the statically applied load at that point. Figure 2 shows the plot of the transverse displacement at the location of the moving load at two levels of foundation stiffness and moving load velocity. The predicted results by the proposed model as well as those of Lee [77] are demonstrated with dashed and solid lines, respectively. According to Figure 2, there is reasonably good agreement between the obtained results by the proposed model and those of Lee [77]. In the case of $v=0.5 V_{c r}$ and $k_{s}=$ $10^{6}$, the discrepancies between the predicted results of the present work and those of Lee [77] are somehow more obvious with respect to other cases, because both inertial effects of the moving load and shear deformation of the beam were taken into account in the proposed model by Lee [77], whereas such effects were not considered in the present study. Thereby, the maximum transverse displacement in the study of Lee [77] is somewhat greater than that predicted by the present model.

In another comparative work, the obtained results by the proposed model are checked against those of Lou et al. [78]. Using finite element method, Lou et al. [78] investigated the transverse vibration of a Timoshenko beam subjected to a moving mass. Consider an elastic beam with the properties given by Lou et al. [78] and define $D_{d}=\frac{\max \{u(l / 2, t)\}}{\delta}$ and $S=v / V_{c r}$, where the definition of $\delta$ and $\stackrel{\delta}{V}_{c r}$ is given in the previous part. In 


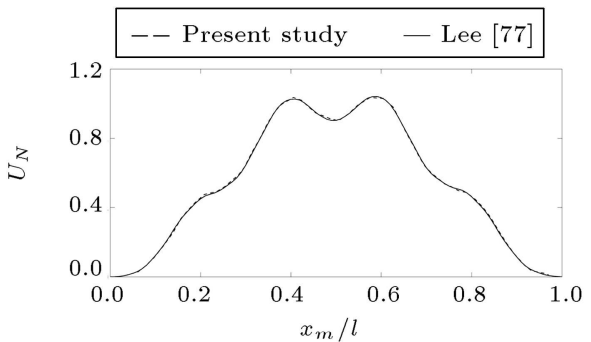

(a)

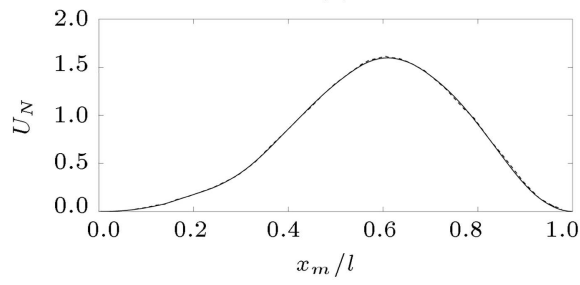

(c)

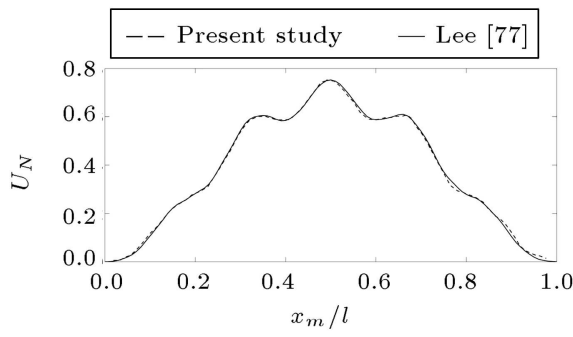

(b)

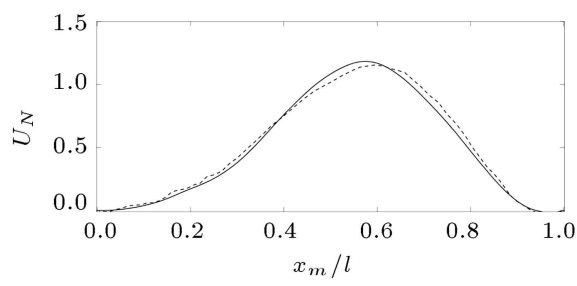

(d)

Figure 2. Normalized transverse displacement at the location of the moving load in terms of its normalized location: (a) $v=0.11 V_{c r}$ and $k_{s}=0$, (b) $v=0.11 V_{c r}$ and $k_{s}=10^{6},(\mathrm{c}) v=0.5 V_{c r}$ and $k_{s}=0,(\mathrm{~d}) v=0.5 V_{c r}$ and $k_{s}=10^{6}$.

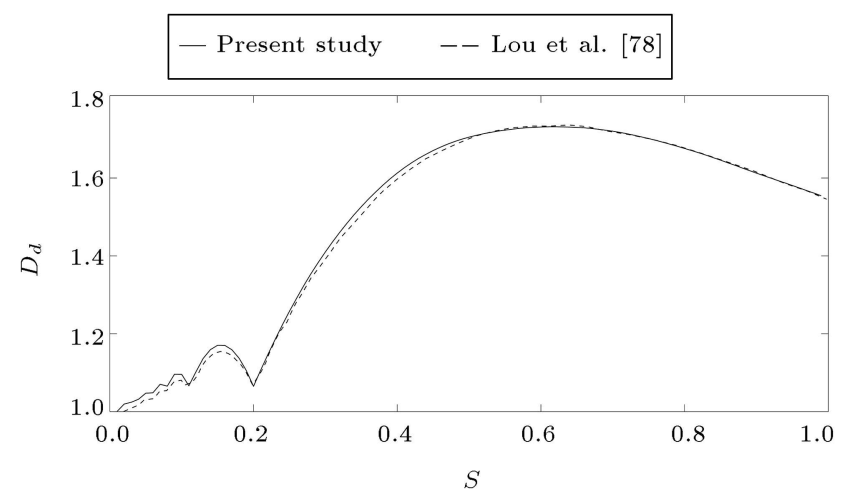

Figure 3. The plotted results of $D_{d}$ as a function of $S$.

Figure 3, the plots of $D_{d}$ in terms of $S$ are demonstrated based on the proposed model and the model of Lou et al. [78]. In a wide range of velocities of the moving mass, the present model can obtain the results of Lou et al. [78] with good accuracy, as illustrated in Figure 3. Accordingly, the maximum deflection commonly increases with the velocity of the moving load up to $v=0.6 V_{c r}$. For velocities greater than this value, both of the above-mentioned models predict a reduction in the maximum transverse displacement of the beam as the velocity of the moving load increases. In the following section, the poroelastic beam is analyzed to explore how variations in the moving load velocity can affect the maximum elastic field of the skeleton as well as the maximum pore pressure of the fluid phase.

\subsection{Parametric studies}

By using the proposed model, the effects of moving velocity, permeability of the porous medium, stiffness of the foundation, and viscosity of the pore fluid on dynamic displacement and pore pressure moment as well as their maximum values are to be examined. In all calculations, $P=1000 \mathrm{~N}, A=0.5 \mathrm{~m}^{2}$, and $l=5 \mathrm{~m}$ were considered for two porous structures with low and high permeability coefficients. The mechanical properties of these two porous media are given in Table 1 . In this table, $k^{s}, k^{f}$, e, and $\nu$ represent the solid bulk modulus, fluid bulk modulus, void ratio, and Poisson's ratio, respectively, and we have $\eta=1-\frac{k_{m}}{k^{s}}$ and $\beta=\frac{\eta-\phi}{k^{s}}+\frac{\phi}{k^{f}}$ where $k_{m}=\frac{E}{3(1-2 \nu)}$ and $\phi=\frac{e}{1+e}$. The permeability coefficients of the considered less and more permeable media in order are related to the void ratio by:

$$
k=10^{\frac{e}{0.45}-10}\left(\frac{\mathrm{cm}}{\mathrm{s}}\right),
$$

and:

$$
k=2.4622\left(\frac{0.01 e^{3}}{1+e}\right)^{0.7825}\left(\frac{\mathrm{cm}}{\mathrm{s}}\right)
$$

Table 1. The mechanical properties of two poroelastic structures.

\begin{tabular}{lcccccccc}
\hline & \multicolumn{7}{c}{ Porous medium } \\
\cline { 2 - 8 } & $E\left(\frac{\mathrm{N}}{\mathrm{m}^{2}}\right)$ & $k^{s}\left(\frac{\mathrm{N}}{\mathrm{m}^{2}}\right)$ & $k^{f}\left(\frac{\mathrm{N}}{\mathrm{m}^{2}}\right)$ & $k_{s}\left(\frac{\mathrm{N}}{\mathrm{m}^{2}}\right)$ & $\mu_{f}($ Pa.s $)$ & $\rho\left(\frac{\mathrm{kg}}{\mathrm{m}^{3}}\right)$ & $e$ & $\nu$ \\
\cline { 2 - 8 } Low permeable & $2 \times 10^{7}$ & $20.9 \times 10^{9}$ & $2.29 \times 10^{9}$ & $27 \times 10^{6}$ & $10^{-3}$ & 1750 & 0.6 & 0.4 \\
High permeable & $5 \times 10^{7}$ & $20 \times 10^{9}$ & $2.3 \times 10^{9}$ & $37 \times 10^{6}$ & $10^{-3}$ & 1950 & 0.45 & 0.3 \\
\hline
\end{tabular}




\subsubsection{Dynamic responses at different velocity levels of the moving load}

In Figurs 4 and 5, the time history plots of the normalized displacement and pore pressure moment of the poroelastic beam's midspan point, namely $W_{N}$ and $M_{p N}$, are provided at different velocity levels of the moving load. These normalized values are defined by:

$$
W_{N}=\frac{w(l / 2, t)}{w_{s t, \max }} \quad \text { and } \quad M_{p N}=\frac{M_{p}(l / 2, t)}{M_{s t, \max }},
$$

where $w_{s t, \max }$ and $M_{s t \text {, max }}$ are the maximum values of deflection and bending moment of the drained poroelastic beam, respectively, subjected to the statically pointed load at its midspan point. The results associated with the less and more permeable structures are specified by $(a)$ and $(b)$, respectively. In these plots, the graphs which are demonstrated by the solid, dashed, dashed-dotted, and dotted lines are pertinent to the velocities $50,70,100$, and $120 \frac{\mathrm{m}}{\mathrm{s}}$, respectively. Figures 4(a) and 5(a) display that the maximum values of both displacement and pore pressure moment increase as the moving load velocity generally increases from 50 to $70 \frac{\mathrm{m}}{\mathrm{s}}$; however, in the velocity range of $100-120 \frac{\mathrm{m}}{\mathrm{s}}$, these parameters would experience a reduction. It is implied that for the less permeable medium, the variations of displacement as well as pore pressure moment as a function of velocity follow an identical trend. For the more permeable medium (see

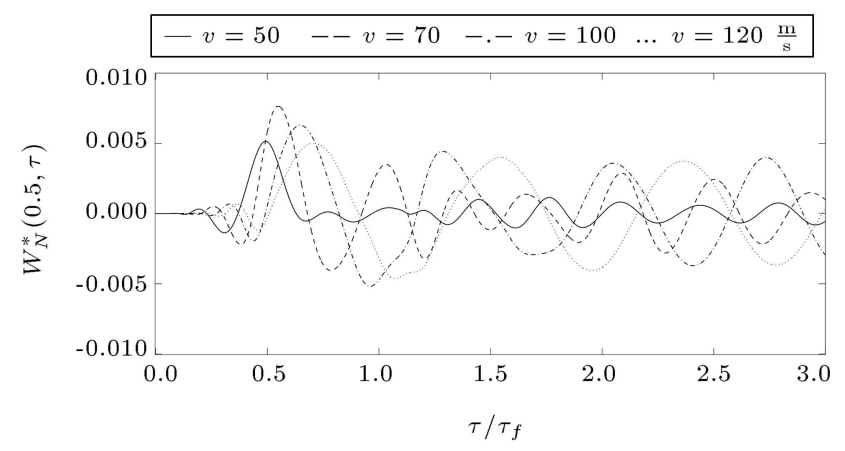

(a)

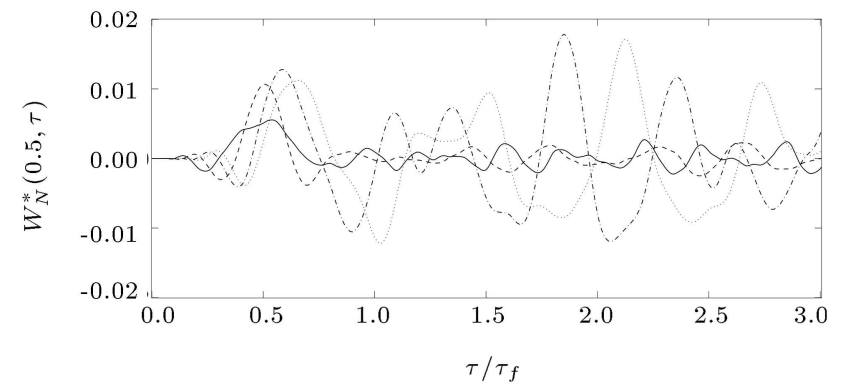

(b)

Figure 4. Time history plots of the normalized transverse displacement of the midspan point of the poroelastic beam at various levels of the moving load velocity: (a) Less permeable structure and (b) more permeable structure.

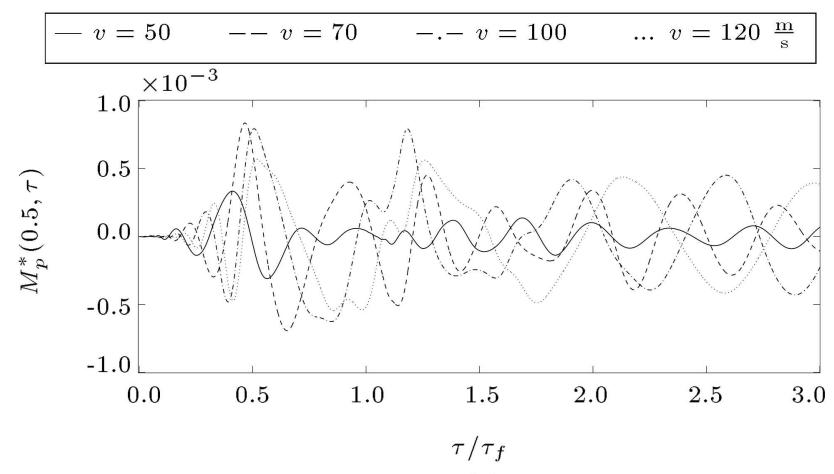

(a)

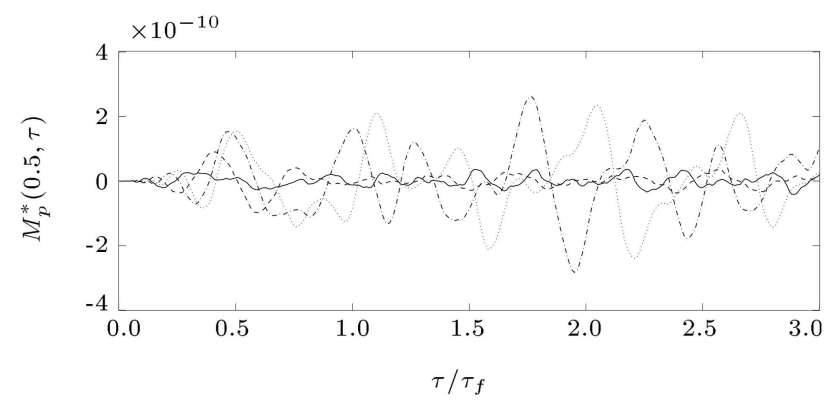

(b)

Figure 5. Time history plots of the normalized pore pressure moment of the midspan point of the poroelastic beam at different velocity levels of the moving load: (a) Less permeable structure and (b) more permeable structure.

Figures 4(b) and 5(b)), the maximum values of both displacement and pore pressure moment increase as the velocity of the moving load increases up to $100 \frac{\mathrm{m}}{\mathrm{s}}$. For velocity levels greater than this value, the maximum displacement and pore pressure moment decrease as the moving load velocity increases. Both Figures 4 and 5 show that the peaks of the plots of both dynamic displacement and pore pressure moment move from the first phase of excitation to the second one as the moving load velocity increases. Additionally, at higher velocity levels of the moving load, dissipation of the amplitudes of both dynamic displacement and pore pressure moment takes a longer amount of time.

\subsubsection{Influence of the moving load velocity on the maximum dynamic responses}

Another interesting aim of this study is to investigate the effect of moving load velocity on the maximum values of transverse displacement and pore pressure moment. In different conditions, analysis was carried out at different levels of permeability coefficient and foundation stiffness of the poroelastic beam. Figure 6(a) and (b) show variations in the maximum dynamic displacement as a function of the velocity of the moving load for two considered media and at three levels of the foundation stiffness (i.e., $k_{s}, 1.5 k_{s}$, and $2 k_{s}$ ). According to the stiffness of the foundation, the 


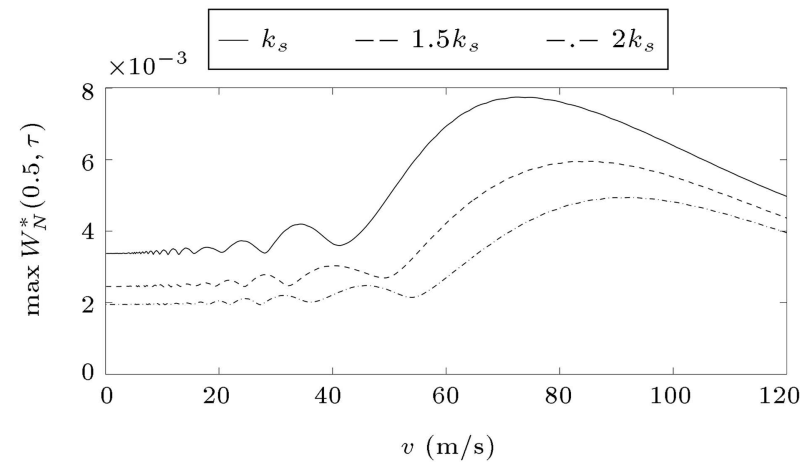

(a)

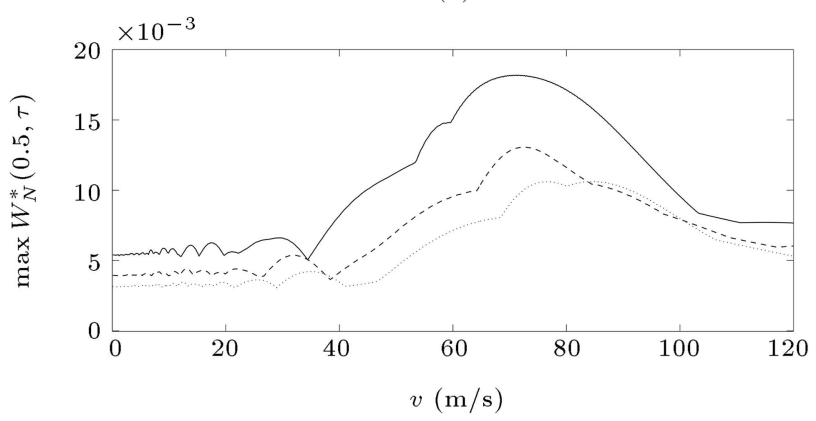

(b)

Figure 6. Maximum normalized transverse displacement of the midspan point of the poroelastic beam in terms of the moving load velocity for different values of the foundation stiffness: (a) Less permeable structure and (b) more permeable structure.

maximum transverse displacement commonly increases up to a certain level as the moving load velocity grows. Increase in the foundation stiffness would lead to an increase in velocity. However, for velocities greater than the defined value, the maximum transverse displacement is generally reduced due to the moving load velocity. For instance, the corresponding velocities of the peak points for $k_{s}, 1.5 k_{s}$, and $2 k_{s}$ in order are 73,85 , and $90 \frac{\mathrm{m}}{\mathrm{s}}$ for the low permeable medium, and 71,73 , and $84 \frac{\mathrm{m}}{\mathrm{s}}$ for the more permeable structure. Based on these data, in the case of the less permeable structure, the influence of the foundation stiffness on the variation of the above-mentioned velocity becomes clear, as compared to the more permeable medium. For both less and more permeable structures, the maximum dynamic displacement and pore pressure moment are generally reduced as the foundation stiffness increases (see Figure 6(a) and (b)). For the less permeable poroelastic beam, the peak of the plot is reduced by about 23 and $16 \%$ considering the transition from $k_{s}$ to $1.5 k_{s}$ and then from $1.5 k_{s}$ to $2 k_{s}$, respectively. Considering the increased foundation stiffness in the case of a more permeable structure, the amounts of reduction in order are approximately equal to 28 and $18 \%$. Further, at high levels of foundation stiffness, the influence of foundation stiffness on the maximum dynamic displacement is reduced.

\subsubsection{Influence of the permeability coefficient on the maximum displacement}

The variations in the maximum dynamic displacement of the poroelastic beam in terms of moving load velocity are plotted in Figure $7(\mathrm{a})$ and (b) for different values of the permeability coefficient. The plotted results are given for both less and more permeable media at three levels of the permeability coefficient for each structure. According to the given results, irrespective of the permeability coefficient, the maximum dynamic displacement experiences an increase up to a certain level as the velocity of the moving load decreases for both poroelastic structures. Thereafter, the maximum dynamic displacement is attenuated as the moving load velocity increases. According to Figure 7(a), increasing the permeability coefficient of the less permeable structure leads to an increase in the maximum transverse displacement at all velocity levels of the moving load. This phenomenon becomes more obvious for velocity levels greater than $60 \frac{\mathrm{m}}{\mathrm{s}}$. However, Figure $7(\mathrm{~b})$ displays that the increase of the permeability coefficient of the more permeable structure has fairly no effect on the maximum transverse displacement.

The effect of the permeability coefficient of the poroelastic medium on the maximum pore pressure moment is of particular interest. For this purpose, the plots of maximum pore pressure moment as a function of moving load velocity at three levels of permeability

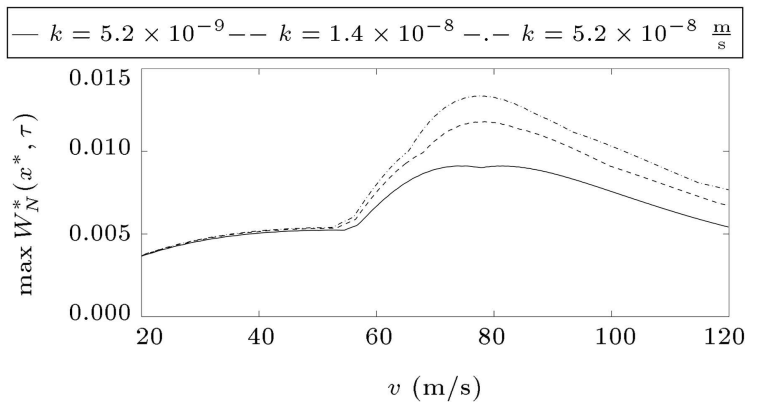

(a)

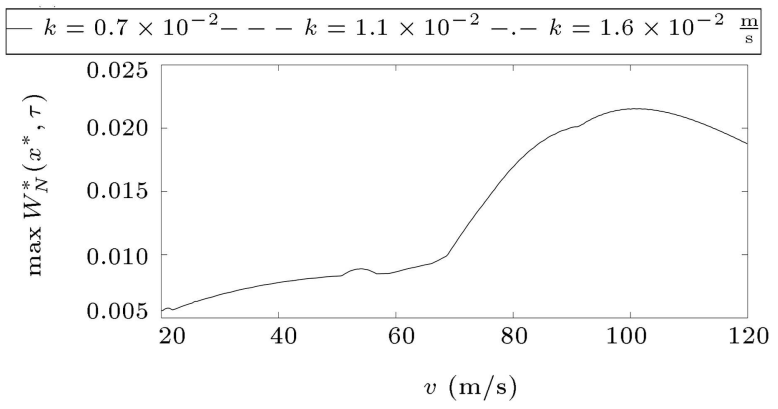

(b)

Figure 7. Influence of the moving load velocity on the maximum normalized transverse displacement of the poroelastic beam for different permeability coefficients: (a) Less permeable structure and (b) more permeable structure. 
coefficient are given in Figure $8(\mathrm{a})$ and (b). These plots show that the maximum pore pressure moment increases with the moving load velocity up to a certain level. For a velocity rate of the moving load greater than that certain value, the maximum value of the pore pressure moment is reduced with the velocity of the moving load. A scrutiny of the depicted results reveals that the variation of the permeability coefficient has insignificant effect on variations in the velocity of the moving load. Additionally, the maximum pore pressure moment is reduced as the permeability coefficient increases. This phenomenon holds at all considered velocity levels of the moving load. For instance, for the less permeable structure, with an increase in the permeability coefficient from $5.2 \times 10^{-9}$ to $1.4 \times 10^{-8}$ and then from $1.4 \times 10^{-8}$ to $5.2 \times 10^{-8} \frac{\mathrm{m}}{\mathrm{s}}$, the peak of the plot of the maximum pore pressure moment is reduced by about 60 and $56 \%$, respectively. Additionally, for the poroelastic beam made of more permeable materials, by increasing the permeability coefficient from $0.7 \times 10^{-2}$ to $1.1 \times 10^{-2}$ and then from $1.1 \times 10^{-2}$ to $1.6 \times 10^{-2} \frac{\mathrm{m}}{\mathrm{s}}$, the peak of the plot of the maximum pore pressure moment in order decreases by about 37 and $31 \%$. Such results also indicate that variations in the maximum pore pressure in terms of permeability would follow at a lower rate

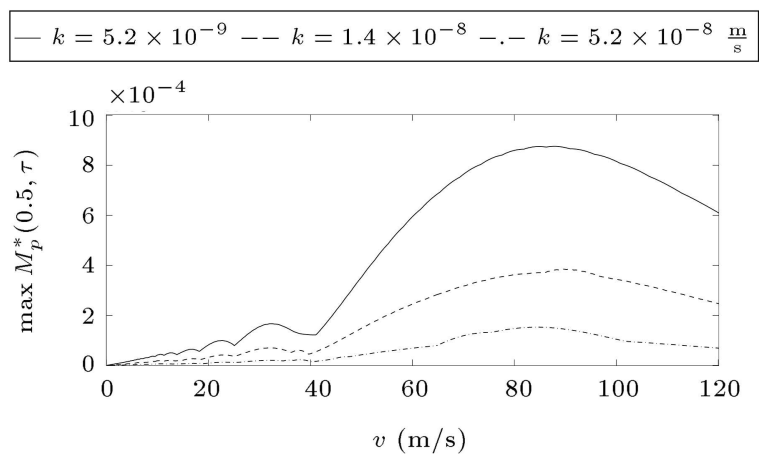

(a)

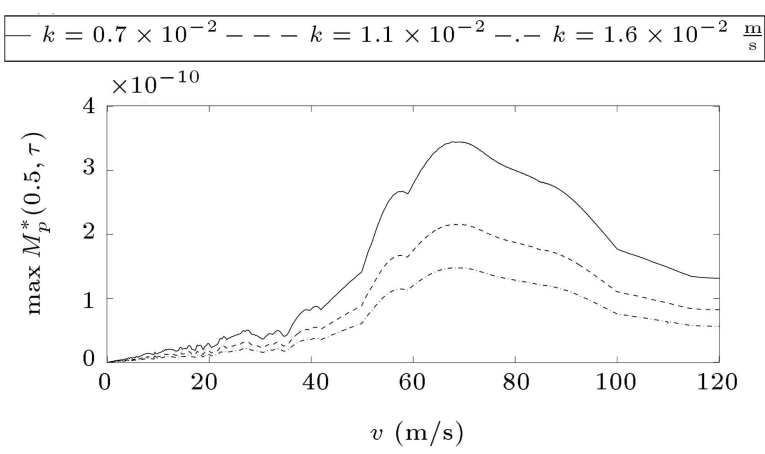

(b)

Figure 8. Influence of the moving load velocity on the maximum dimensionless pore pressure moment of the poroelastic beam for different permeability coefficients: (a) Less permeable structure (b) more permeable structure. as the permeability coefficient of the poroelastic beam increases.

\subsubsection{Influence of the foundation stiffness and pore fluid viscosity on the maximum displacement}

An attempt was made to find out how stiffness of the poroelastic foundation and pore fluid viscosity could affect the maximum dynamic response of the poroelastic beam. To this end, the maximum transverse displacement predicted by the proposed model as a function of the foundation stiffness ratio, $k_{f}=k s / k_{s 0}$, is plotted in Figure $9(\mathrm{a})$ and (b) (note: $k_{s 0}$ is the initially considered value of the foundation stiffness as stated in Table 1). These figures respectively correspond to the less and more permeable structures. According to Figure 9(a) and (b), the maximum dynamic displacement is reduced as the foundation stiffness increases. Such a reduction is more apparent at lower levels of the foundation stiffness. Interestingly, by increasing the pore fluid viscosity, the maximum transverse displacement decreases. Such a behavior is more apparent for the less permeable beam with respect to the more permeable one, because at lower levels of the permeability coefficient, by increasing the viscosity, the fluid movement inside the pores slows down. Such an interesting behavior could be interpreted through Eq. (8) using the definition of the factor, $k$.

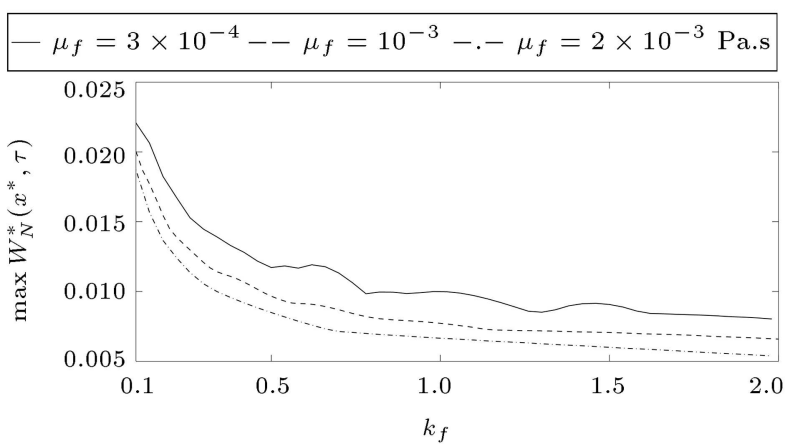

(a)

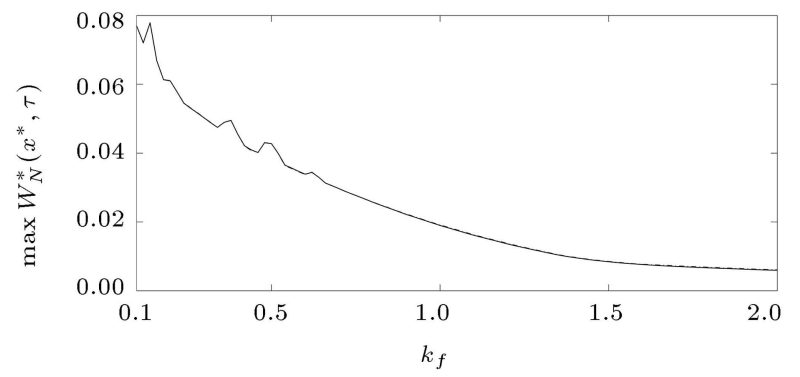

(b)

Figure 9. Influence of the foundation's stiffness on the maximum normalized transverse displacement of the poroelastic beam for different values of the viscosity of the pore fluid: (a) Less permeable structure and (b) more permeable structure. 


\subsubsection{Influence of the void ratio on the maximum pore pressure moment}

The effect of the porosity on the generated maximum displacement of the poroelastic beam due to the moving load is the subject of another crucial study that should be explored.

For this purpose, the plots of maximum dynamic displacement as a function of void ratio for both less and more permeable structures are given in Figure 10(a) and (b). The plotted results are provided for three levels of the pore fluid viscosity (i.e., $\mu_{f}=$ $3 \times 10^{-4}, 10^{-3}$, and $2 \times 10^{-3}$ Pa.s). According to the demonstrated results in Figure 10(a) and (b), the maximum dynamic displacement is reduced as the void ratio of the poroelastic beam increases. The main reason why this phenomenon occurs is rooted in the given relations of the permeability coefficient in the less and more permeable media. Based on these relations, as the void ratio increases from 0.6 to 0.95 for the less permeable medium and from 0.4 to 0.6 for the more permeable medium, the permeability coefficient of these structures increases from $5.2 \times 10^{-9}$ to $5.2 \times$ $10^{-8}$ and from $0.7 \times 10^{-2}$ to $1.6 \times 10^{-2}$, respectively. As explained in an earlier part, the maximum dynamic responses are generally reduced as the permeability coefficient of the porous medium increases. Moreover, the effect of the pore fluid viscosity on the poroelastic beam at lower levels of void ratio becomes clearer.

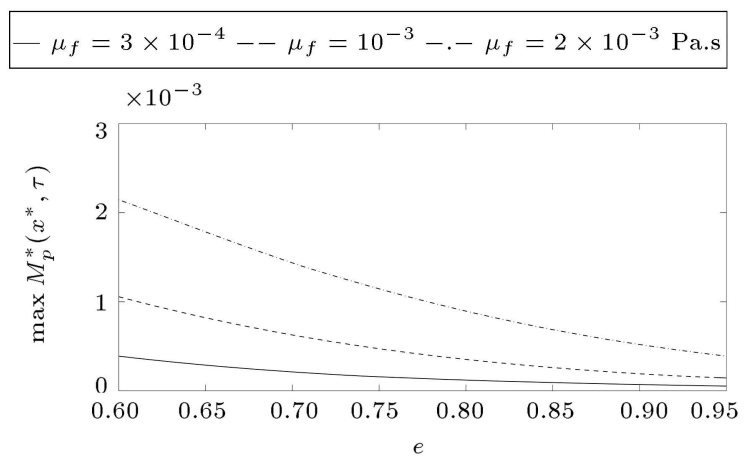

(a)

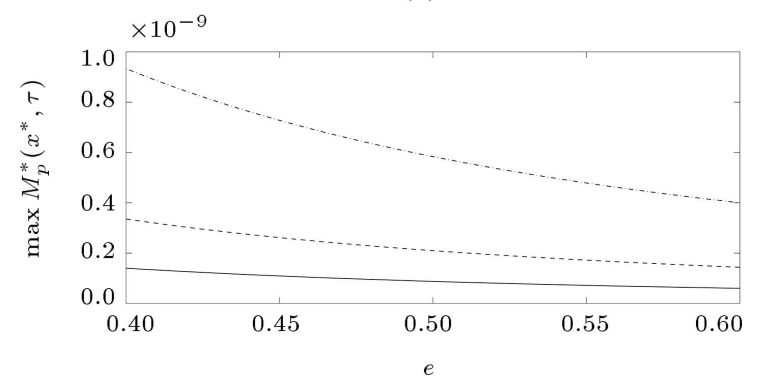

(b)

Figure 10. Influence of the void ratio on the maximum normalized pore pressure moment of the poroelastic beam for different values of the viscosity of the pore fluid: (a) Less permeable structure and (b) more permeable structure.

\section{Conclusions}

This study investigated lateral vibrations of an elastically rested isotropic poroelastic beam with the longitudinal movement of pore fluid under the pressure of a moving pointed load. To this end, the beam was simulated in accordance with the hypotheses of the continuum-based Euler-Bernoulli beam theory and the interaction of the beam structure and its underlying medium was modeled by the Winkler foundation. By implementing Biot's poroelastic theory, the governing equations were extracted carefully and then solved by using the assumed mode method in the case of simply supported boundary conditions. The explicit expressions of the dynamic transverse displacement and the pore pressure were derived using Laplace transform technique. The influences of the moving load velocity, permeability coefficient, foundation stiffness, pore fluid viscosity, and porosity on the maximum dynamic displacement as well as the pore pressure were addressed in some detail.

The suggested methodology in the present work used for obtaining vibrations of poroelastic beamlike structures provides a solid base to perform the dynamic analysis of more complex structures including poroelastic media subjected to moving loads allowing fluid movement in bi-direction, multiple-poroelasticsystems under the action of moving loads, and so on. These critical subjects are regarded as hot topics for future works that could be followed by interested investigators.

\section{References}

1. Terzaghi, K. "Die Berechnung der Durchassigkeitsziffer des Tones aus dem Verlauf der hydrodynamischen Spannungsercheinungen, Sitzungsberichte der Akademie der Wissenschaften in Wien, MathematischNaturwissenschaftliche Klasse [The calculation of permeability of clay from the variation of hydrodynamic stress with time, Proceedings of the Academy of Sciences in Vienna, School of Mathematical Sciences], 2A, 132, pp. 125-138 (1932).

2. Biot, M.A. "General theory of three-dimensional consolidation", Journal of Applied Physics, 12, pp. 155164 (1941).

3. Biot, M.A. "Theory of elasticity and consolidation for a porous anisotropic solid", Journal of Applied Physics, 26, pp. 182-185 (1955).

4. Biot, M.A. "Generalized theory of acoustic propagation in porous dissipative media", The Journal of the Acoustical Society of America, 34, pp. 1254-1264 (1962).

5. Biot, M.A. "Mechanics of deformation and acoustic propagation in porous media", Journal of Applied Physics, 33, pp. 1482-1498 (1962). 
6. Selvadurai, A.P.S. "Mechanics of poroelastic media", Solid Mechanics and Its Application, 35, Kluwer (1996).

7. Wang, H.F., Theory of Linear Poroelasticity with Applications to Geomechanics and Hydrogeology, Princeton University Press (2000).

8. Nowinski, J.L. and Davis, C.F. "A model of the human skull as a poroelastic spherical shell subjected to a quasistatic load", Mathematical Biosciences, 8, pp. 397-416 (1970).

9. Nowinski, J.L. and Davis, C.F. "The flexure and torsion of bones viewed as anisotropic poroelastic bodies", International Journal of Engineering Science, 10, pp. 1063-1079 (1972).

10. Manfredini, P., Cocchetti, G., Maier, G., Redaelli, A., and Montevecchi, F.M. "Poroelastic finite element analysis of a bone specimen under cyclic loading", Journal of Biomechanics, 32, pp. 135-144 (1999).

11. Lu, J.F., Jeng, D.S., and Lee, T.L. "Dynamic response of a piecewise circular tunnel embedded in a poroelastic medium", Soil Dynamics and Earthquake Engineering, 27, pp. 875-891 (2007).

12. Cai, Y., Cao, Z., Sun, H., and Xu, C. "Effects of the dynamic wheel-rail interaction on the ground vibration generated by a moving train", International Journal of Solids and Structures, 47, pp. 2246-2259 (2010).

13. Cao, Z. and Boström, A. "Dynamic response of a poroelastic half-space to accelerating or decelerating trains", Journal of Sound and Vibration, 332, pp. 2777-2794 (2013).

14. Lo, W.C. "Decoupling of the coupled poroelastic equations for quasistatic flow in deformable porous media containing two immiscible fluids", Advances in Water Resources, 29, pp. 1893-1900 (2006).

15. Lo, W.C., Sposito, G., and Majer, E. "Immiscible two-phase fluid flows in deformable porous media", Advances in Water Resources, 25, pp. 1105-1117 (2002).

16. Coussy, O. "Poromechanics of freezing materials", Journal of Mechanics and Physics of Solids, 53, pp. 1689-1718 (2005).

17. Yamamoto, T., Maruyama, S., Nishiwaki, S., and Yoshimura, M. "Topology design of multi-material soundproof structures including poroelastic media to minimize sound pressure levels", Computer Methods in Applied Mechanics and Engineering, 198, p. 14391455 (2009).

18. Hou, P.F., Zhao, M., Tong, J., and Fu, B. "Threedimensional steady-state Green's functions for fluidsaturated, transversely isotropic, poroelastic bimaterials", Journal of Hydrology, 496, pp. 217-224 (2013).

19. Zhou, J., Bhaskar, A., and Zhang, X. "Sound transmission through a double-panel construction lined with poroelastic material in the presence of mean flow", Journal of Sound and Vibration, 332, pp. 3724-3734 (2013).

20. Boutin, C. "Behavior of poroelastic isotropic beam derivation by asymptotic expansion method", Journal of Mechanics and Physics of Solids, 60, pp. 1063-1087 (2012).

21. Carcione, J.M. and Gerardo, Q.G. "Some aspects of the physics and numerical modeling of Biot compressional waves", Journal of Computational Acoustics, 3, pp. 261-280 (1995).

22. White, J.E. "Computed seismic speeds and attenuation in rocks with partial gas saturation", Geophysics, 40, pp. 224-232 (1975).

23. Mandel, J. "Consolidation des sols (étude mathématique)", [Consolidation of soils (mathematical analysis)], Geotechnique, 3, pp. 287-299 (1953).

24. Cryer, C.W. "A comparison of the three-dimensional consolidation theories of Biot and Terzaghi", Quarterly Journal of Mechanics and Applied Mathematics, 16, pp. 401-412 (1963).

25. Schiffman, R.L., Chen, A.T.F., and Jordan, J.C. "An analysis of consolidation theories", Journal of the Soil Mechanics and Foundations Division, 95, pp. 295-312 (1969).

26. Gibson, R.E., Gobert, A., and Schiffman, R.L. "On Cryer's problem with large displacements and variable permeability", Geotechnique, 40, pp. 627-631 (1990).

27. Abousleiman, Y., Cheng, A.H.D., Cui, L., Detournay, E., and Roegiers, J.C. "Mandel's problem revisited", Geotechnique, 46, pp. 187-195 (1996).

28. Li, L.P., Schulgasser, K., and Cederbaum, G. "Theory of poroelastic beams with axial diffusion", Journal of Mechanics and Physics of Solids, 43, pp. 2023-2042 (1995).

29. Cederbaum, G., Schulgasser, K., and Li, L.P. "Interesting behavior patterns of poroelastic beams and columns", International Journal of Solids and Structures, 35, pp. 4931-4943 (1998).

30. Li, L.P., Schulgasser, K., and Cederbaum, G. "Buckling of poroelastic columns with axial diffusion", International Journal of Mechanical Sciences, 39, pp. 409415 (1997).

31. Olsson, M. "On the fundamental moving load problem", Journal of Sound and Vibration, 145, pp. 299307 (1991).

32. Savin, E. "Dynamic amplification factor and response spectrum for the evaluation of vibrations of beams under successive moving loads", Journal of Sound and Vibration, 248, pp. 267-288 (2001). 
33. Michaltsos, G.T. "Dynamic behaviour of a single-span beam subjected to loads moving with variable speeds", Journal of Sound and Vibration, 258, pp. 359-372 (2002).

34. Kiani, K. "Nonlocal continuum-based modeling of a nanoplate subjected to a moving nanoparticle. Part I: Theoretical formulations", Physica E: Lowdimensional Systems and Nanostructures, 44, pp. 229248 (2011).

35. Kiani, K. "Nonlocal continuum-based modeling of a nanoplate subjected to a moving nanoparticle. Part II: Parametric studies", Physica E: Low-dimensional Systems and Nanostructures, 44, pp. 249-269 (2011).

36. Kiani, K. "Vibrations of biaxially tensioned-embedded nanoplates for nanoparticle delivery", Indian Journal of Science and Technology, 6, pp. 4894-4902 (2013).

37. Ebrahimzadeh Hassanabadi, M., Amiri, J.V., and Davoodi, M.R. "On the vibration of a thin rectangular plate carrying a moving oscillator", Scientia Iranica, 21, pp. 284-294 (2014).

38. Lee, U. "Revisiting the moving mass problem: on set of separation between the mass and beam", Journal of Vibration and Acoustics, ASME, 118, pp. 516-521 (1996).

39. Lee, H.P. "The dynamic response of a Timoshenko beam subjected to a moving mass", Journal of Sound and Vibration, 198, pp. 249-256 (1996).

40. Michaltsos, G., Sophianopoulos, D., and Kounadis, A.N. "The effect of a moving mass and other parameters on the dynamic response of a simply supported beam", Journal of Sound and Vibration, 191, pp. 357362 (1996).

41. Ichikawa, M., Miyakawa, Y., and Matsuda, A. "Vibration analysis of the continuous beam subjected to a moving mass", Journal of Sound and Vibration, 230, pp. $493-506$ (2000).

42. Kiani, K., Nikkhoo, A., and Mehri, B. "Prediction capabilities of classical and shear deformable beam theories excited by a moving mass", Journal of Sound and Vibration, 320, pp. 632-648 (2009).

43. Kiani, K., Nikkhoo, A., and Mehri, B. "Parametric analyses of multispan viscoelastic shear deformable beams under excitation of a moving mass", Journal of Vibration and Acoustics, ASME, 131, p. 051009 (2009).

44. Azam, S.E., Mofid, M., and Khoraskani, R.A. "Dynamic response of Timoshenko beam under moving mass", Scientia Iranica, 20, pp. 50-56 (2013).

45. Nikkhoo, A., Rofooei, F.R., and Shadnam, M.R. "Dynamic behavior and modal control of beams under moving mass", Journal of Sound and Vibration, 306, pp. $712-724$ (2007).
46. Hassanabadi, M.E., Nikkhoo, A., Amiri, J.V., and Mehri, B. "A new orthonormal polynomial series expansion method in vibration analysis of thin beams with non-uniform thickness", Applied Mathematical Modelling, 37, pp. 8543-8556 (2013).

47. Ahmadi, M. and Nikkhoo, A. "Utilization of characteristic polynomials in vibration analysis of nonuniform beams under a moving mass excitation", Applied Mathematical Modelling, 38, pp. 2130-2140 (2014).

48. Nikkhoo, A. "Investigating the behavior of smart thin beams with piezoelectric actuators under dynamic loads", Mechanical Systems and Signal Processing, 45, pp. 513-530 (2014).

49. Nikkhoo, A., Farazandeh, A., Hassanabadi, M.E., and Mariani, S. "Simplified modeling of beam vibrations induced by a moving mass by regression analysis", Acta Mechanica, 226, pp. 2147-2157 (2015).

50. Nikkhoo, A., Farazandeh, A., and Hassanabadi, M.E. "On the computation of moving mass/beam interaction utilizing a semi-analytical method", Journal of the Brazilian Society of Mechanical Sciences and Engineering, 38, pp. 761-771 (2016).

51. Khoraskani, R.A., Mofid, M., Azam, S.E., and Ebrahimzadeh Hassanabadi, M. "A new simplified formula in prediction of the resonance velocity for multiple masses traversing a thin beam", Scientia Iranica, 23, pp. 133-141 (2016).

52. Azam, S.E., Mofid, M., and Khoraskani, R.A. "Dynamic response of Timoshenko beam under moving mass", Scientia Iranica A: Transactions in Civil Engineering, 20, pp. 50-56 (2013).

53. Rofooei, F.R., Enshaeian, A., and Nikkhoo, A. "Dynamic response of geometrically nonlinear, elastic rectangular plates under a moving mass loading by inclusion of all inertial components", Journal of Sound and Vibration, 394, pp. 497-514 (2017).

54. Hassanabadi, M.E., Attari, K.A., Nikkhoo, A., and Mariani, S. "Resonance of a rectangular plate influenced by sequential moving masses", Coupled Systems Mechanics, 5, pp. 87-100 (2016).

55. Lin, Y.H. and Trethewey, M.W. "Finite element analysis of elastic beams subjected to moving dynamic loads", Journal of Sound and Vibration, 136, pp. 323342 (1990).

56. Yang, Y.B. and Yau, J.D. "Vehicle-bridge interaction element for dynamic analysis", Journal of Structural Engineering, ASCE, 123, pp. 1512-1518 (1997).

57. Yang, Y.B. and Wu, Y.S. "A versatile element for analyzing vehicle-bridge interaction response", Engineering Structures, 23, pp. 452-469 (2001).

58. Kiani, K., Avili, H.G., and Kojorian, A.N. "On the role of shear deformation in dynamic behavior of a fully saturated poroelastic beam traversed by a moving load", International Journal of Mechanical Sciences, 94, pp. 84-95 (2015). 
59. Pak, R.Y.S. and Guzina, B.B. "Three-dimensional Green's functions for a multilayered half-space in displacement potentials", Journal of Engineering Mechanics, ASCE, 128, pp. 449-461 (2002).

60. Theodorakopoulos, D.D. "Dynamic analysis of a poroelastic half-plane soil medium under moving loads", Soil Dynamics and Earthquake Engineering, 23, pp. 521-533 (2003).

61. Jin, B., Yue, Z.Q., and Tham, L.G. "Stresses and excess pore pressure induced in saturated poroelastic halfspace by moving line load", Soil Dynamics and Earthquake Engineering, 24, pp. 25-33 (2004).

62. Cai, Y., Sun, H., and Xu, C. "Steady state responses of poroelastic half-space soil medium to a moving rectangular load", International Journal of Solids and Structures, 44, p. 71837196 (2007).

63. Lu, J.F. and Jeng, D.S. "A half-space saturated poroelastic medium subjected to a moving point load", International Journal of Solids and Structures, 44, pp. 573-586 (2007).

64. Sun, H., Cai, Y., and Xu, C. "Three-dimensional simulation of track on poroelastic halfspace vibrations due to a moving point load", Soil Dynamics and Earthquake Engineering, 30, pp. 958-967 (2010).

65. Cao, Z., Cai, Y., Boström, A., and Zheng, J. "Semianalytical analysis of the isolation to moving-load induced ground vibrations by trenches on a poroelastic half-space", Soil Dynamics and Earthquake Engineering, 331, pp. 947-961 (2012).

66. Shi, L., Sun, H., Cai, Y., Xu, C., and Wang, P. "Validity of fully drained, fully undrained and u$\mathrm{p}$ formulations for modeling a poroelastic half-space under a moving harmonic point load", Soil Dynamics and Earthquake Engineering, 42, pp. 292-301 (2012).

67. Etchessahar, M., Sahraoui, S., and Brouard, B. "Bending vibrations of a rectangular poroelastic plate", Comptes Rendus de l'Academie des Sciences-Series IIB-Mechanics, 329, pp. 615-620 (2001).

68. Theodorakopoulos, D.D. and Beskos, D.E. "Flexural vibrations of poroelastic plates", Acta Mechanica, 103, pp. 191-203 (1994).

69. Horoshenkov, K.V. and Sakagami, K. "A method to calculate the acoustic response of a thin, baffled, simply supported poroelastic plate", The Journal of the Acoustical Society of America, 110, pp. 904-917 (2001).

70. Leclaire, P., Horoshenkov, K.V., and Cummings, A. "Transverse vibrations of a thin rectangular porous plate saturated by a fluid", Journal of Sound and Vibration, 247, pp. 1-18 (2001).

71. Yahia, S.A., Atmane, H.A., Houari, M.S.A., and Tounsi, A. "Wave propagation in functionally graded plates with porosities using various higher-order shear deformation plate theories", Structural Engineering and Mechanics, 53, pp. 1143-1165 (2015).
72. Reddy, P.M. and Tajuddin, M. "Exact analysis of the plane-strain vibrations of thickwalled hollow poroelastic cylinders", International Journal of Solids and Structures, 37, pp. 3439-3456 (2000).

73. Tajuddin, M. and Shah, S.A. "On torsional vibrations of infinite hollow poroelastic cylinders", Journal of Mechanics of Materials and Structures, 2, pp. 189-200 (2007).

74. Bourada, M., Kaci, A., Houari, M.S.A., and Tounsi, A. "A new simple shear and normal deformations theory for functionally graded beams", Steel and Composite Structures, 18, pp. 409-423 (2015).

75. Belabed, Z., Houari, M.S.A., Tounsi, A., Mahmoud, S.R., and Anwar Beg, O. "An efficient and simple higher order shear and normal deformation theory for functionally graded material (FGM) plates", Composites Part B-Engineering, 60, pp. 274-283 (2014).

76. Mahi, A., Adda Bedia, E.A., and Tounsi, A. "A new hyperbolic shear deformation theory for bending and free vibration analysis of isotropic, functionally graded, sandwich and laminated composite plates", Applied Mathematical Modeling, 39, pp. 2489-2508 (2015).

77. Lee, H.P. "Dynamic response of a Timoshenko beam on a Winkler foundation subjected to a moving mass", Applied Acoustics, 55, pp. 203-215 (1998).

78. Lou, P., Dai, G.L., and Zeng, Q.Y. "Finite-element analysis for a Timoshenko beam subjected to a moving mass", Proceedings of the Institution of Mechanical Engineers Part C: Journal of Mechanical Engineering Science, 220, pp. 669-678 (2006).

Appendix A. Evaluation of $A_{i n}^{\prime}, B_{i n}^{\prime}, A_{i n}^{\prime \prime}$, and $B_{i n}^{\prime \prime}$

Appendix A1. When the moving load is in contact with the base poroelastic beam In this case, Eq. (20a) can be rewritten as follows:

$$
\begin{aligned}
& \mathcal{L}\left\{a_{n}\right\}= \\
& \frac{\sum_{i=1}^{5}\left(\Re\left(A_{i_{n}}^{\prime}\right)+\mathrm{i} \Im\left(A_{i_{n}}^{\prime}\right)\right)\left(s^{4}-F_{i_{n}}^{\prime} s^{3}+G_{i_{n}}^{\prime} s^{2}-H_{i_{n}}^{\prime} s+L_{i_{n}}^{\prime}\right)}{\prod_{i=1}^{5}\left(s-p_{i_{n}}^{\prime}\right)},
\end{aligned}
$$

where:

$$
\begin{aligned}
F_{i_{n}}^{\prime} & =\sum_{j=1, j \neq i}^{5} p_{j_{n}}^{\prime}, \quad G_{i_{n}}^{\prime}=\sum_{j=1, j \neq i}^{5} \sum_{k=j+1, k \neq i}^{5} p_{j_{n}}^{\prime} p_{k_{n}}^{\prime}, \\
H_{i_{n}}^{\prime} & =\sum_{j=1, j \neq i}^{5} \sum_{k=j+1, k \neq i}^{5} \sum_{l=k+1, l \neq i}^{5} p_{j_{n}}^{\prime} p_{k_{n}}^{\prime} p_{l_{n}}^{\prime}, \\
L_{i_{n}}^{\prime} & =\prod_{j=1, j \neq i}^{5} p_{j_{n}}^{\prime} ; \quad i=1,2, \cdots, 5
\end{aligned}
$$




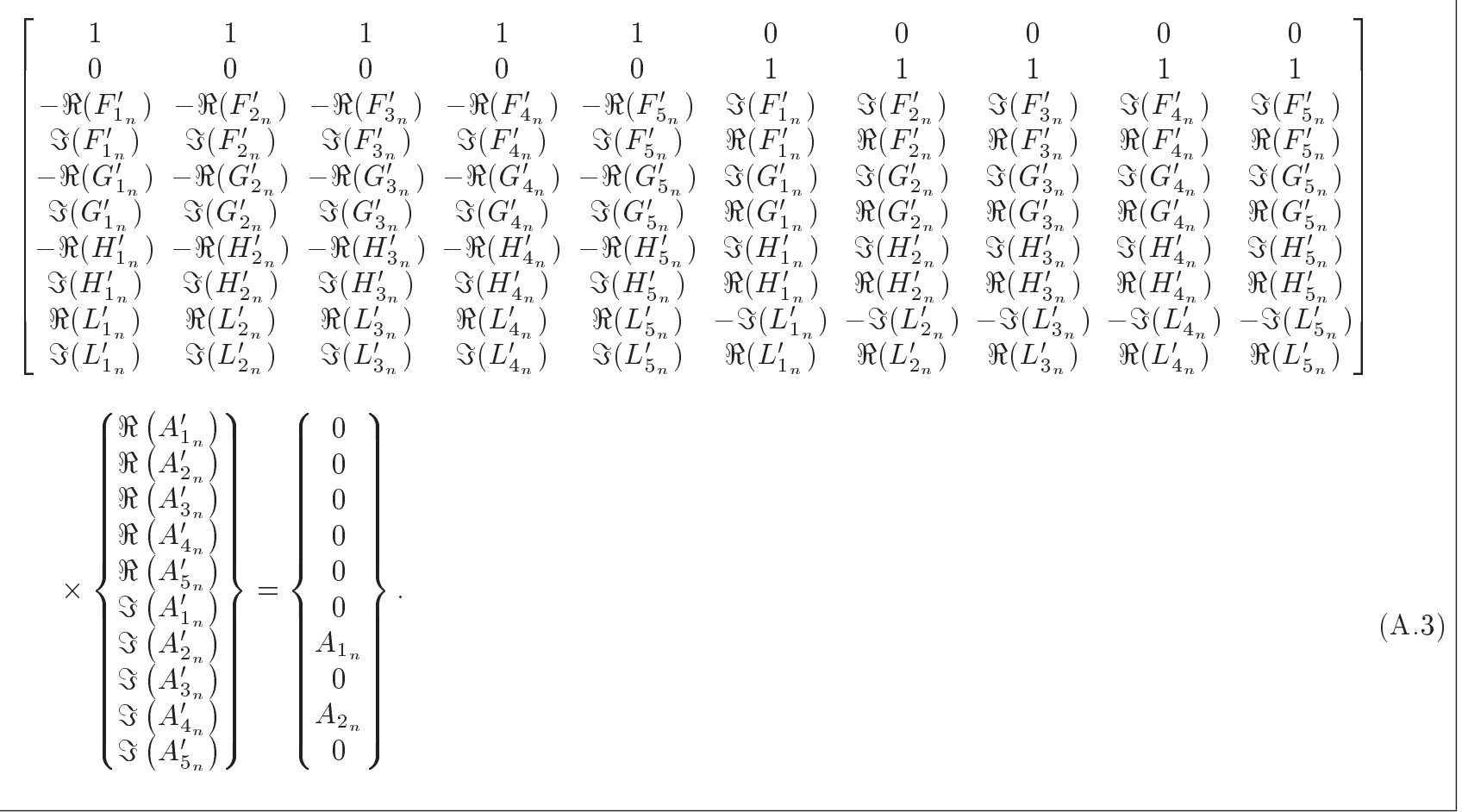

Box A.I

$$
\left[\begin{array}{cccccc}
1 & 1 & 1 & 0 & 0 & 0 \\
0 & 0 & 0 & 1 & 1 & 1 \\
-\Re\left(F_{1_{n}}^{\prime \prime}\right) & -\Re\left(F_{2_{n}}^{\prime \prime}\right) & -\Re\left(F_{3_{n}}^{\prime \prime}\right) & \Im\left(F_{1_{n}}^{\prime \prime}\right) & \Im\left(F_{2_{n}}^{\prime \prime}\right) & \Im\left(F_{3_{n}}^{\prime \prime}\right) \\
-\Im\left(F_{1_{n}}^{\prime \prime}\right) & -\Im\left(F_{2_{2}}^{\prime \prime}\right) & -\Im\left(F_{3_{n}}^{\prime \prime}\right) & -\Re\left(F_{1_{n}}^{\prime \prime}\right) & -\Re\left(F_{2_{n}}^{\prime \prime}\right) & -\Re\left(F_{3_{n}}^{\prime \prime}\right) \\
\Re\left(G_{1_{n}}^{\prime \prime}\right) & \Re\left(G_{2_{n}}^{\prime \prime}\right) & \Re\left(G_{3_{n}}^{\prime \prime}\right) & -\Im\left(G_{1_{n}}^{\prime \prime}\right) & -\Im\left(G_{2_{n}}^{\prime \prime}\right) & -\Im\left(G_{3_{n}}^{\prime \prime}\right) \\
\Im\left(G_{1_{n}}^{\prime \prime}\right) & \Im\left(G_{2_{n}}^{\prime \prime}\right) & \Im\left(G_{3_{n}}^{\prime \prime}\right) & \Re\left(G_{1_{n}}^{\prime \prime}\right) & \Re\left(G_{2_{n}}^{\prime \prime}\right) & \Re\left(G_{3_{n}}^{\prime \prime}\right)
\end{array}\right] \times\left\{\begin{array}{l}
\Re\left(A_{1_{n}}^{\prime \prime}\right) \\
\Re\left(A_{2_{n}}^{\prime \prime}\right) \\
\Re\left(A_{3_{n}}^{\prime \prime}\right) \\
\Im\left(A_{1_{n}}^{\prime \prime}\right) \\
\Im\left(A_{2_{n}}^{\prime \prime}\right) \\
\Im\left(A_{3_{n}}^{\prime \prime}\right)
\end{array}\right\}=\left\{\begin{array}{l}
\Re\left(A_{1_{n}}^{\prime}\right) \\
\Im\left(A_{1_{n}}^{\prime}\right) \\
\Re\left(A_{2_{n}}^{\prime}\right) \\
\Im\left(A_{2_{n}}^{\prime}\right) \\
\Re\left(A_{3_{n}}^{\prime}\right) \\
\Im\left(A_{3_{n}}^{\prime}\right)
\end{array}\right\} .
$$

Box A.II

By comparing Eq. (A.1) with Eq. (18), Eq. (A.3), as shown in Box A.I, is obtained. By solving the set of linear algebraic equations in Eq. (A.3), the magnitudes of $A_{i_{n}}^{\prime}$ would be readily determined. By taking the inverse Laplace transform of both sides of Eq. (A.1), one can arrive at:

$$
a_{n}(\tau)=\sum_{i=1}^{5}\left(\Re\left(A_{i_{n}}^{\prime}\right)+\mathrm{i} \Im\left(A_{i_{n}}^{\prime}\right)\right) \exp \left(p_{i_{n}}^{\prime} \tau\right) .
$$

In order to calculate the unknown coefficients $B_{i_{n}}^{\prime}$, a procedure identical to that used for evaluating $A_{i_{n}}^{\prime}$ is followed.

\section{Appendix A2. When the moving load has left the poroelastic beam}

During the course of free vibration, Eq. (24a) can be rewritten as:

$$
\begin{aligned}
\mathcal{L} & \left\{a_{n}\left(\tau^{\prime}\right)\right\} \\
= & \frac{\sum_{i=1}^{3}\left(\Re\left(A_{i_{n}}^{\prime \prime}\right)+\mathrm{i} \Im\left(A_{i_{n}}^{\prime \prime}\right)\right)\left(s^{2}-F_{i_{n}}^{\prime \prime} s+G_{i_{n}}^{\prime \prime}\right)}{\prod_{i=1}^{3}\left(s-p_{i_{n}}^{\prime \prime}\right)},
\end{aligned}
$$

where:

$$
\begin{array}{ll}
F_{1_{n}}^{\prime \prime}=p_{2_{n}}^{\prime \prime}+p_{3_{n}}^{\prime \prime}, & F_{2_{n}}^{\prime \prime}=p_{1_{n}}^{\prime \prime}+p_{3_{n}}^{\prime \prime}, \\
F_{3_{n}}^{\prime \prime}=p_{1_{n}}^{\prime \prime}+p_{2_{n}}^{\prime \prime}, & G_{1_{n}}^{\prime \prime}=p_{2_{n}}^{\prime \prime} p_{3_{n}}^{\prime \prime}, \\
G_{2_{n}}^{\prime \prime}=p_{1_{n}}^{\prime \prime} p_{3_{n}}^{\prime \prime}, & G_{3_{n}}^{\prime \prime}=p_{1_{n}}^{\prime \prime} p_{2_{n}}^{\prime \prime},
\end{array}
$$

where $p_{i_{n}}^{\prime \prime}$ represents the roots of the third-order polynomial in the denominator of Eq. (24a). Since Eq. (A.5) is equivalent to Eq. (24a), Eq. (A.7), as shown in Box A.II, is obtained. By solving the set of algebraic equations in Eq. (A.7), the unknown parameters $A_{i_{n}}^{\prime \prime}$ 


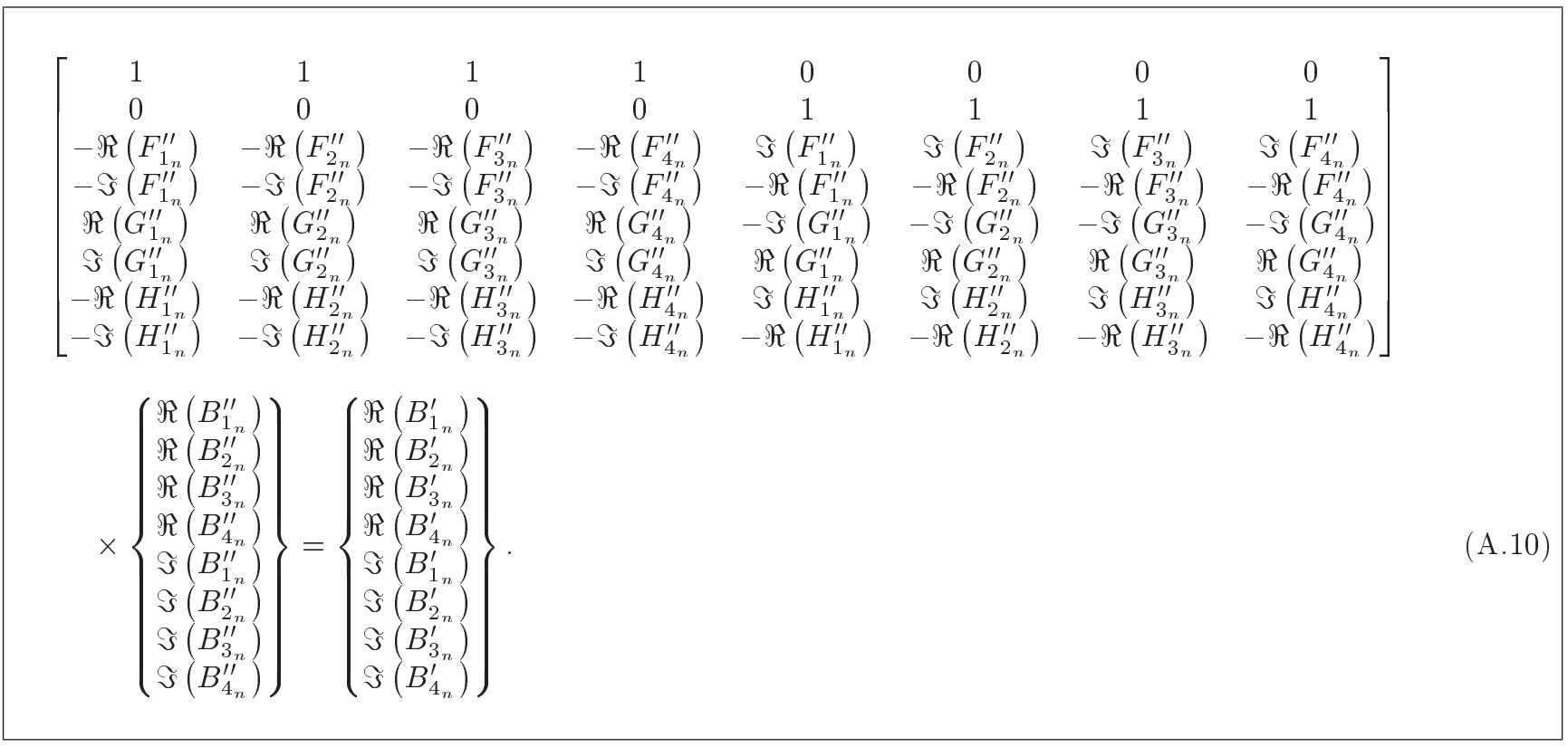

Box A.III

could be determined. Finally, by taking the inverse Laplace transform of Eq. (A.5), the values of $a_{n}\left(\tau^{\prime}\right)$ during the course of free vibration are calculated as follows:

$$
a_{n}\left(\tau^{\prime}\right)=\sum_{i=1}^{3}\left(\Re\left(A_{i_{n}}^{\prime \prime}\right)+\mathrm{i} \Im\left(A_{i_{n}}^{\prime \prime}\right)\right) \exp \left(p_{i_{n}}^{\prime \prime} \tau^{\prime}\right) .
$$

In order to evaluate $b_{n}\left(\tau^{\prime}\right)$, we follow the same procedure mentioned in the previous part. Therefore:

$$
b_{n}\left(\tau^{\prime}\right)=\sum_{i=1}^{4}\left(\Re\left(B_{i_{n}}^{\prime \prime}\right)+\mathrm{i} \Im\left(B_{i_{n}}^{\prime \prime}\right)\right) \exp \left(q_{i_{n}}^{\prime \prime} \tau^{\prime}\right),
$$

where $q_{i_{n}}^{\prime \prime}$ represents the poles of the denominator of Eq. (24b), and the magnitudes of $B_{i_{n}}^{\prime \prime}$ can be determined by solving Eq. (A.10) as shown in Box A.III, where:

$$
\begin{array}{ll}
F_{1_{n}}^{\prime \prime}=q_{1_{n}}^{\prime \prime}+q_{3_{n}}^{\prime \prime}+q_{4_{n}}^{\prime \prime}, & F_{2_{n}}^{\prime \prime}=q_{2_{n}}^{\prime \prime}+q_{3_{n}}^{\prime \prime}+q_{4_{n}}^{\prime \prime}, \\
F_{3_{n}}^{\prime \prime}=q_{1_{n}}^{\prime \prime}+q_{2_{n}}^{\prime \prime}+q_{3_{n}}^{\prime \prime}, & F_{4_{n}}^{\prime \prime}=q_{1_{n}}^{\prime \prime}+q_{2_{n}}^{\prime \prime}+q_{4_{n}}^{\prime \prime},
\end{array}
$$

$$
\begin{aligned}
& G_{1_{n}}^{\prime \prime}=q_{2_{n}}^{\prime \prime} q_{3 n}^{\prime \prime}+q_{2_{n}}^{\prime \prime} q_{4 n}^{\prime \prime}+q_{3_{n}}^{\prime \prime} q_{4 n}^{\prime \prime}, \\
& G_{2_{n}}^{\prime \prime}=q_{1_{n}}^{\prime \prime} q_{3_{n}}^{\prime \prime}+q_{1_{n}}^{\prime \prime} q_{4 n}^{\prime \prime}+q_{3_{n}}^{\prime \prime} q_{4 n}^{\prime \prime}, \\
& G_{3_{n}}^{\prime \prime}=q_{1_{n}}^{\prime \prime} q_{2_{n}}^{\prime \prime}+q_{2_{n}}^{\prime \prime} q_{4 n}^{\prime \prime}+q_{1_{n}}^{\prime \prime} q_{4 n}^{\prime \prime}, \\
& G_{4_{n}}^{\prime \prime}=q_{2 n}^{\prime \prime} q_{3_{n}}^{\prime \prime}+q_{1_{n}}^{\prime \prime} q_{2_{n}}^{\prime \prime}+q_{1_{n}}^{\prime \prime} q_{3 n}^{\prime \prime}, \\
& H_{i_{n}}^{\prime \prime}=\sum_{j=1, j \neq i}^{4} q_{j_{n}}^{\prime \prime} ; \quad i=1,2, \ldots, 4
\end{aligned}
$$

\section{Biographies}

A. Nikkhoo. His/her biography was not available at the time of publication.

R. Tafakor. His/her biography was not available at the time of publication.

M. Mofid. His biography was not available at the time of publication. 